\title{
Comparing different MR angiography strategies of carotid stents in a vascular flow model: toward stent-specific recommendations in MR follow-up
}

\author{
Andreas M. J. Frölich • Sara M. Pilgram-Pastor • \\ Marios N. Psychogios • Alexander Mohr • \\ Michael Knauth
}

Received: 16 May 2010 /Accepted: 27 July 2010 /Published online: 19 August 2010

(C) The Author(s) 2010. This article is published with open access at Springerlink.com

\begin{abstract}
Introduction Carotid artery stenting (CAS) requires adequate follow-up imaging to assess complications such as in-stent stenosis or occlusion. Options include digital subtraction angiography, CT angiography, ultrasound, and MR angiography (MRA), which may offer a non-invasive option for CAS follow-up imaging. The aim of this study was to assess contrast-enhanced MRA (CE-MRA) and three-dimensional time-of-flight MRA (3D-TOF) for visualization of the in-stent lumen in different carotid stents.

Methods In this study, we compared CE-MRA and 3DTOF of five different carotid stents (Guidant Acculink ${ }^{\circledR}$, Cordis Precise ${ }^{\circledR}$, Boston Wallstent ${ }^{\circledR}$, Abbot Vascular Xact $^{\circledR}$, Cook Zilver $\left.{ }^{\circledR}\right)$ in three diameters $(4,6$, and $8 \mathrm{~mm})$ using a vascular flow model at $3.0 \mathrm{~T}$ with the help of a recently developed carotid surface coil. Stent-related artifacts were objectively assessed by calculating artificial lumen narrowing (ALN) and relative in-stent signal (RIS). Results RIS and ALN depended heavily on stent type, stent diameter, and the employed MR sequence. ALN and RIS were relatively favorable for Acculink $^{\circledR}$, Precise $^{\circledR}$, and Zilver ${ }^{\circledR}$ stents with both CE-MRA and 3D-TOF. CE-MRA provided better results for the Wallstent, while the Xact stent was difficult to visualize with both MRA protocols. Conclusion Both CE-MRA and 3D-TOF are viable options for depicting the in-stent lumen in carotid stents. For specific stents, 3D-TOF provided image quality comparable to CEMRA and may thus be suitable for in vivo assessment.
\end{abstract}

A. M. J. Frölich $(\bowtie) \cdot$ S. M. Pilgram-Pastor • M. N. Psychogios •

A. Mohr $\cdot$ M. Knauth

Department of Neuroradiology,

University of Göttingen Medical School,

Robert-Koch-Str. 40,

37075 Göttingen, Germany

e-mail: a.froelich@gmail.com
Development of stent-specific pathways for follow-up imaging seems advisable to address stent-related differences in image quality.

Keywords Three-dimensional time-of-flight angiography Carotid stents · MR angiography · Artifacts .

Contrast-enhanced MR angiography

$\begin{array}{ll}\text { Abbreviation Key } \\ \text { CAS } & \begin{array}{l}\text { Carotid artery stenting } \\ \text { Contrast-enhanced magnetic resonance } \\ \text { CE-MRA }\end{array} \\ \text { CTA } & \begin{array}{l}\text { Computed tomography angiography } \\ \text { Three-dimensional time-of-flight angiography }\end{array} \\ \text { 3D-TOF } & \text { Artificial lumen narrowing } \\ \text { ALN } & \text { Relative in-stent signal } \\ \text { RIS } & \text { Magnetic resonance angiography } \\ \text { MRA } & \text { Digital subtraction angiography } \\ \text { DSA } & \text { Signal intensity } \\ \text { SI } & \text { Diameter } \\ \text { D } & \end{array}$

\section{Introduction}

Carotid artery stenting (CAS) is a widely used method for prevention of stroke in individuals with symptomatic or highgrade carotid artery stenosis. To assess treatment effectiveness and reliably detect possible complications such as restenosis or vessel occlusion, adequate follow-up is mandatory. Options include Doppler ultrasound, digital subtraction angiography (DSA), CT angiography (CTA), and magnetic resonance angiography (MRA). While DSA and CTA require the use of ionizing radiation, ultrasound is user-dependant and may offer limited reproducibility. In comparison, MRA offers the 
advantage of being non-invasive and free from ionizing radiation, making it a promising option for follow-up imaging. Stent-related artifacts, however, currently limit its wide-spread diagnostic use. Previous studies on MRA in the presence of vascular stents focused on contrast-enhanced MRA (CEMRA). For CE-MRA, it has been shown that the in-stent lumen can be visualized at different field strengths [1-9]. However, stent-related artifacts leading to artificial lumen narrowing (ALN) and in-stent signal attenuation limit lumen visibility. Furthermore, CE-MRA requires the use of gadolinium contrast agents, which carry the risk of allergic reactions and renal complications, including nephrogenic systemic fibrosis $[10,11]$ in patients with coexisting renal disease. Time-of-flight angiography (TOF) is a wellestablished and widely used sequence type for depicting intra- and extracranial vascular structures that do not require the use of contrast agents. To date, no clear guidelines exist for follow-up examinations after CAS [12]. To help develop standard protocols for follow-up imaging, different modalities need to be objectively compared regarding ALN, in-stent signal attenuation, and stent-related artifacts. Previous studies on stent imaging employed CE-MRA, CT angiography, and DSA [1-9]. To our knowledge, no investigations exist to date that systematically analyze lumen visibility and stentrelated artifacts with three-dimensional TOF angiography (3D-TOF). With high-field MRI and surface coils, powerful tools have been developed to shorten acquisition time and improve image quality. This may help overcome weaknesses traditionally associated with TOF angiography, making it a promising sequence type for stent imaging. The purpose of this study was to assess the effectiveness and reliability of 3D-TOF in depicting the intra-stent lumen and to evaluate its potential use in follow-up imaging after CAS, focusing especially on differences to CE-MRA.

\section{Materials and methods}

Vascular phantom

A vascular phantom was constructed using a hollow plastic cylinder filled with ultrasound gel (LIBOmed, Kleve, Germany). Silicon tubes (neoLab GmbH, Heidelberg, Germany) were used as vessels. Stents were placed inside the tubes, and the system was filled with normal saline solution. For CE-MRA, contrast medium was added at a dilution of 1:200 (Gadovist, Bayer Schering Pharma AG, Berlin, Germany). Flow was achieved by connecting a cardiovascular pump (3M Delphin II centrifugal pump, 3M, St. Paul, MN, USA). Flow was pulsatile, with flow volume set to $400 \mathrm{ml} / \mathrm{min}$, approximating in vivo conditions for the common carotid artery [13]. Five different stent types (Guidant Acculink ${ }^{\circledR}$, Cordis Precise ${ }^{\circledR}$, Boston Wallstent ${ }^{\circledR}$,
Abbot Vascular Xact ${ }^{\circledR}$, and Cook Zilver ${ }^{\circledR}$ ) were deployed in tubes with diameters of 4,6 , and $8 \mathrm{~mm}$. Table 1 gives an overview of the stents used in this study.

\section{MR imaging}

All MR images were obtained on a Siemens Trio 3.0-T scanner (Siemens Medical Solutions, Erlangen, Germany). A carotid surface coil (Machnet B.V., Eelde, The Netherlands) was used for all sequences in this study. The coil was attached to the plastic cylinder, which functioned as a neck phantom containing the silicone vessels. The vascular flow phantom was placed inside the MR scanner with the longitudinal axis of the stented vessel segment roughly parallel to $B_{0}$. Sequence parameters for 3D-TOF and CEMRA are summarized in Table 2. To illustrate stent geometry (Fig. 1), dynamic flat-panel detector images were obtained (Siemens Axiom Artis Flat Panel, Siemens Medical Solutions, Erlangen, Germany).

Image analysis

Image analysis was performed using the free medical imaging software Osiris (http://www.dim.hcuge.ch/osiris/ 01_Osiris_Presentation_EN.htm). Coronal multiplanar reformations were used for both 3D-TOF and CE-MRA. In-stent signal attenuation was assessed by calculating the relative in-stent signal (RIS) inside the stent. This was achieved by obtaining mean values of signal intensity (SI) from six regions of interest (ROI) placed in the visible tube lumen inside (at least $2 \mathrm{~mm}$ away from the stent wall) and outside the stent ( $\mathrm{SI}_{\mathrm{in} \text {-stent }}$ and $\mathrm{SI}_{\text {tube }}$, respectively). ROIs were placed manually and centered on the stent axis. For all measurements, ROIs were placed at least $1 \mathrm{~cm}$ away from the stent ends because of strong artifacts occurring in the distal stent regions. The RIS was calculated accordingly:

$\operatorname{RIS}(\%)=\left(\mathrm{SI}_{\text {in-stent }} / \mathrm{SI}_{\text {tube }}\right) \times 100 \%$.

The mean and standard deviation were obtained for the resulting three RIS values for each stent and a paired Student's $t$ test was used to compare differences between CE-MRA and 3D-TOF for the RIS values of all sizes of each stent. The level of statistical significance was set at 0.01 .

Visible lumen diameter and ALN were determined in a manner similar to previous investigations [5]: For objective evaluation, signal intensity profiles were obtained along the $x$-axis (perpendicular to the stent axis) both inside the midsegment of the stent and outside the stent. The mean of the SI values of first 10 pixels along this profile (outside the stent) was defined as the SI of the background gel ( $\left.\mathrm{SI}_{\text {gel }}\right)$. In 
Table 1 Overview of stents included in this study

\begin{tabular}{|c|c|c|c|c|c|c|}
\hline Stent name & $\begin{array}{l}\text { Name used in } \\
\text { figures/tables }\end{array}$ & $\begin{array}{l}\text { Stent diameter/ } \\
\text { length }(\mathrm{mm})\end{array}$ & $\begin{array}{l}\text { Tube } \\
\text { diameter } \\
(\mathrm{mm})\end{array}$ & $\begin{array}{l}\text { Stent } \\
\text { design }\end{array}$ & Material & Manufacturer \\
\hline \multirow[t]{3}{*}{ Zilver $^{(\mathbb{R}}$} & Zil8 & $9 / 40$ & 8 & \multirow[t]{3}{*}{ Open cell } & \multirow[t]{3}{*}{ Nitinol } & \multirow{3}{*}{$\begin{array}{l}\text { Cook Vascular Incorporated, } \\
\text { Vandergrift, PA, USA }\end{array}$} \\
\hline & Zil6 & $7 / 40$ & 6 & & & \\
\hline & Zil4 & $5 / 40$ & 4 & & & \\
\hline \multirow[t]{3}{*}{ Acculink $^{(}{ }^{\circledR}$} & Acc 8 & $9 / 30$ & 8 & \multirow[t]{3}{*}{ Open cell } & \multirow[t]{3}{*}{ Nitinol } & \multirow{3}{*}{$\begin{array}{l}\text { Guidant, part of Boston Scientific } \\
\text { Corporate, Natick, MA, USA }\end{array}$} \\
\hline & Acc6 & $7 / 30$ & 6 & & & \\
\hline & Acc 4 & $5 / 30$ & 4 & & & \\
\hline \multirow[t]{3}{*}{ Precise ${ }^{\mathbb{R}}$} & Pre8 & $9 / 30$ & 8 & \multirow[t]{3}{*}{ Open cell } & \multirow[t]{3}{*}{ Nitinol } & \multirow{3}{*}{$\begin{array}{l}\text { Cordis Corporation, Bridgewater, } \\
\text { NJ, USA }\end{array}$} \\
\hline & Pre6 & $7 / 30$ & 6 & & & \\
\hline & Pre4 & $5 / 30$ & 4 & & & \\
\hline \multirow[t]{3}{*}{ Carotid Wallstent ${ }^{\circledR}$} & Wal8 & $10 / 24$ & 8 & \multirow[t]{3}{*}{ Closed cell } & \multirow[t]{3}{*}{ Cobalt alloy } & \multirow{3}{*}{$\begin{array}{l}\text { Boston Scientific Corporate, } \\
\text { Natick, MA, USA }\end{array}$} \\
\hline & Wal6 & $8 / 29$ & 6 & & & \\
\hline & Wal4 & $6 / 22$ & 4 & & & \\
\hline \multirow[t]{2}{*}{$\operatorname{Xact}^{\circledR}$} & Xac8 & $9 / 30$ & 8 & \multirow[t]{2}{*}{ Closed cell } & \multirow[t]{2}{*}{ Nitinol } & \multirow{2}{*}{$\begin{array}{l}\text { Abbot Vascular, Redwood City, } \\
\text { CA, USA }\end{array}$} \\
\hline & Xac6 & $7 / 30$ & 6 & & & \\
\hline
\end{tabular}

a pixel profile analysis, the lumen was defined as all pixels with more than twice the SI of $\mathrm{SI}_{\text {gel }}$. The number of "lumen pixels" along the profiles inside and outside the stent represents the in-stent lumen diameter ( $\left.D_{\text {in-stent }}\right)$ and tube lumen diameter $\left(D_{\text {tube }}\right)$, respectively. Artificial lumen narrowing was then expressed as

$$
\operatorname{ALN}(\%)=\left(D_{\text {tube }}-D_{\text {in }- \text { stent }}\right) / D_{\text {tube }} \times 100 \% \text {. }
$$

A higher ALN value therefore correlates with a smaller apparent lumen diameter on the images and thus with worse lumen visibility. Given the pixel-by-pixel analysis, no mean or standard deviations were obtained for ALN values. This method of ALN calculation was chosen to adequately compare stent appearance under experimental conditions, with the assumption that differences in-stent strut thickness were negligible.

Table 2 Overview of sequence parameters

\begin{tabular}{lcc}
\hline & 3D-TOF & CE-MRA \\
\hline TR (ms) & 27 & 5.26 \\
TE (ms) & 2.98 & 1.57 \\
Flip angle & $35^{\circ}$ & $35^{\circ}$ \\
Matrix size & 256 & 256 \\
Field of view & 200 & 200 \\
Slice thickness (mm) & 0.8 & 0.8 \\
Bandwidth (pixel) & 400 & 320 \\
\hline
\end{tabular}

\section{Results}

In-stent signal attenuation

The RIS differed markedly between stent and sequence types as well as stent diameters (Fig. 2, Table 3). The RIS for the Wallstent ${ }^{\circledR}$ was markedly higher for CE-MRA than for 3D-TOF (44\% vs. $9 \%$ on average for all sizes, $p<0.001$ for each size). In comparison, the average differences for the Acculink ${ }^{\circledR}$ (79\% for CE-MRA vs. $79 \%$ for 3D-TOF for all sizes), Precise (40\% vs. $44 \%)$, and Zilver ${ }^{\circledR}$ stents $(85 \%$ vs. 77\%) were not significant. Finally, the Xact ${ }^{\circledR}$ stent showed very low intraluminal signal with both sequences (RIS $<11 \%$ for CE-MRA, $<4 \%$ for $3 \mathrm{D}-\mathrm{TOF}$ ) and as a result had poor lumen visibility. With the exception of the $6 \mathrm{~mm}$ Zilver $^{\circledR}$ and $6 \mathrm{~mm}$ Acculink ${ }^{\circledR}$, all stents showed higher RIS values with larger stent diameters.

\section{Artificial lumen narrowing}

For the Acculink ${ }^{\circledR}$, Precise ${ }^{\circledR}$, and Zilver ${ }^{\circledR}$ stents, ALN was relatively low $(<25 \%)$ and roughly comparable for CE-MRA and 3D-TOF sequences (Fig. 3). The mean ALN for this group of stents was $17.6 \%$ with CE-MRA and $25.3 \%$ with $3 \mathrm{D}-\mathrm{TOF}$ (average for all stent sizes), although marked variations occurred between stent types and sizes. The Wallstent ${ }^{\circledR}$ showed similarly low ALN values $(<30 \%)$ only for the 6and $8-\mathrm{mm}$ diameters with CE-MRA. The 4-mm stent and all 3D-TOF acquisitions had ALN values $>60 \%$, consistent with poor lumen visibility. Finally, the $\mathrm{Xact}^{\circledR}$ stent showed an almost complete in-stent signal void, and ALN was close to $100 \%$ on average for both CE-MRA, and 3D-TOF. 
Fig. 1 Stent appearance. Images were obtained from $\mathrm{Zil}$, Acc8, Pre8, Wal8, and Xac8 stents. DYNA represents a volumetric coronal reconstruction from a dynamic flat-panel detector $\mathrm{CT}$ acquisition of the stents. $3 D-T O F$ indicates coronal images from the three-dimensional time-offlight angiography study, and $C E-M R A$ represents coronal images of contrast-enhanced magnetic resonance angiography performed for the respective stents

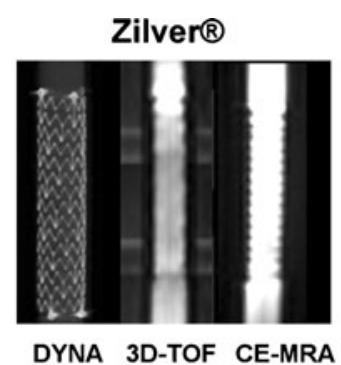

\section{Wallstent ${ }^{\circledR}$}

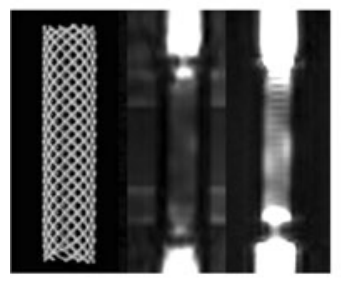

DYNA 3D-TOF CE-MRA

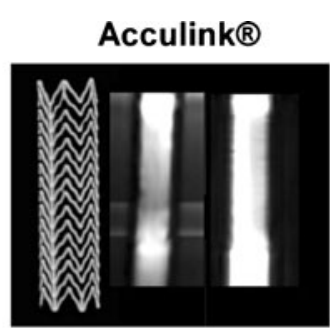

DYNA 3D-TOF CE-MRA

Xact ${ }^{\circledR}$

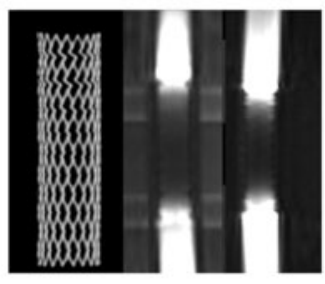

DYNA 3D-TOF CE-MRA

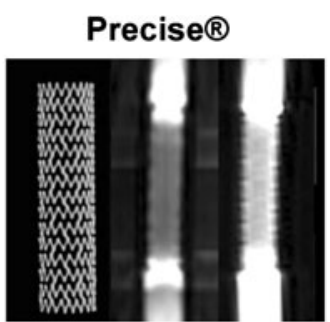

DYNA 3D-TOF CE-MRA

\section{Discussion}

\section{Artifact types}

Appearance of stents with 3D-TOF and CE-MRA is shown in Fig. 1 for the 8-mm stents. Two major types of stentrelated artifacts are described in the literature: susceptibility artifacts and radiofrequency artifacts [6-8]. Susceptibility artifacts are caused by the different magnetic susceptibility of the stent material and the tissue around the stent. Radiofrequency artifacts, on the other hand, result from so-called eddy currents induced inside the stent struts, shielding out the radiofrequency pulse and thus diminishing signal strength. In this study, overall artifact patterns were comparable between the two sequence types used, although there were differences in the degree of observed artifacts. The three artifact patterns most commonly observed in this study were a strong signal gain or loss at the stent ends, a signal void surrounding the stent struts and a homogenous reduction of the in-stent-signal. Previous investigators have pointed out that the strong artifacts encountered with stainless steel-based stents may be related to susceptibility artifacts, whereas the differences in artifacts for cobalt alloy and nitinol-based stents may primarily be based on different degrees of radiofrequency shielding [4, 14].

The degree of artifacts and subsequent changes in lumen visibility, ALN, and RIS varied markedly depending on stent type, size, and sequences used. Interestingly, although RIS was generally higher for larger stent diameters (with few exceptions), there was no likewise correlation for ALN, although it was observed by other authors [15]. This may in part be due to the different ways ALN and RIS were obtained: RIS calculation included data from the homogenous signal at least $2 \mathrm{~mm}$ away from the stent struts, whereas the ALN was calculated from the SI profile across the entire stent diameter, including the more inhomogeneous regions
Fig. 2 Relative in-stent signal. Bars represent RIS values for CE-MRA (dark gray bars) and 3D-TOF (light gray bars) for each stent in the study. Standard deviation is indicated by thin bars

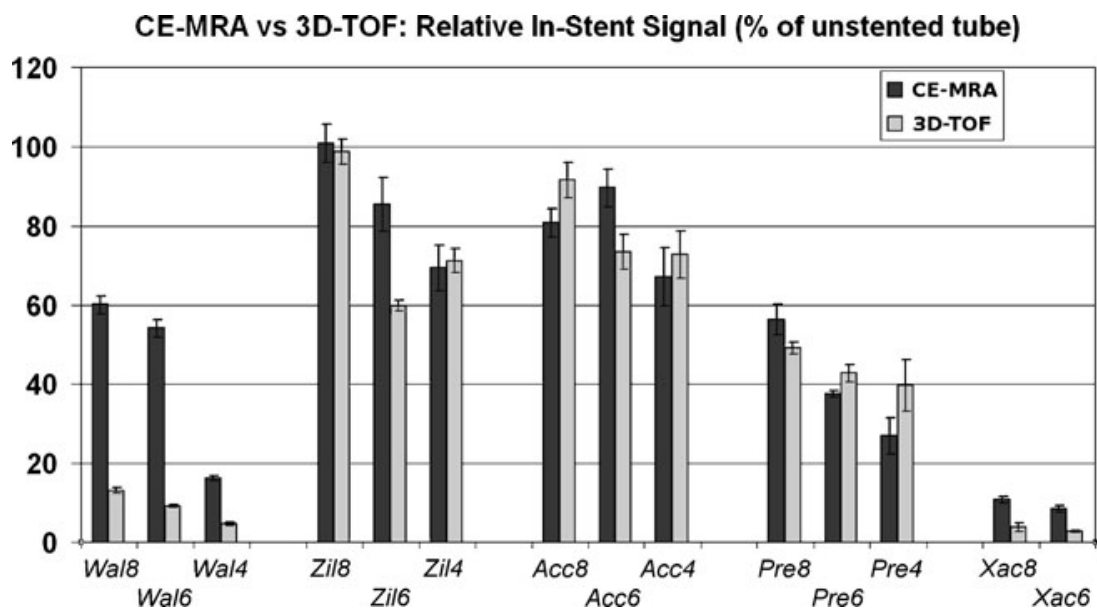


Table 3 Detailed results for relative in-stent signal (RIS) and artificial lumen narrowing (ALN)

\begin{tabular}{|c|c|c|c|c|c|}
\hline & $\begin{array}{l}\text { Average RIS }(\%) \\
\text { for CE-MRA }\end{array}$ & $\begin{array}{l}\text { Average RIS }(\%) \\
\text { for } 3 \mathrm{D}-\mathrm{TOF}\end{array}$ & $\begin{array}{l}p \text { value (RIS CE-MRA } \\
\text { vs. } 3 \mathrm{D}-\mathrm{TOF} \text { ) }\end{array}$ & $\begin{array}{l}\text { ALN (\%) for } \\
\text { CE-MRA }\end{array}$ & $\begin{array}{l}\text { ALN (\%) for } \\
\text { 3D-TOF }\end{array}$ \\
\hline Wal8 & 60.0 & 13.2 & 0.0006 & 25.0 & 60.0 \\
\hline Wal6 & 54.1 & 9.1 & 0.0005 & 30.0 & 100.0 \\
\hline Wal4 & 16.3 & 4.7 & 0.0003 & 75.0 & 100.0 \\
\hline Wallstent ${ }^{\circledR}$ average & 43.5 & 9.0 & & 43.3 & 86.7 \\
\hline Zil8 & 100.8 & 98.7 & 0.3154 & 8.3 & 30.0 \\
\hline Zi16 & 85.4 & 59.7 & 0.0158 & 20.0 & 25.0 \\
\hline Zil4 & 69.4 & 71.1 & 0.3763 & 37.5 & 20.0 \\
\hline Zilver ${ }^{(\mathbb{B}}$ average & 85.2 & 76.5 & & 21.9 & 25.0 \\
\hline Acc8 & 80.8 & 91.5 & 0.0508 & 0.0 & 20.0 \\
\hline Acc6 & 89.6 & 73.4 & 0.0439 & 0.0 & 25.0 \\
\hline Acc 4 & 67.0 & 72.7 & 0.2691 & 25.0 & 20.0 \\
\hline Acculink $^{\circledR}$ average & 79.2 & 79.2 & & 8.3 & 21.7 \\
\hline Pre8 & 56.3 & 49.2 & 0.0647 & 0.0 & 30.0 \\
\hline Pre6 & 37.5 & 42.8 & 0.0161 & 30.0 & 37.5 \\
\hline Pre4 & 26.9 & 39.7 & 0.0633 & 37.5 & 20.0 \\
\hline Precise ${ }^{\circledR}$ average & 40.3 & 43.9 & & 22.5 & 29.2 \\
\hline Xac8 & 10.7 & 3.9 & 0.0126 & 91.7 & 100.0 \\
\hline Xac6 & 8.4 & 2.8 & 0.0059 & 100.0 & 100.0 \\
\hline Xact $^{\circledR}$ average & 9.6 & 3.3 & & 95.8 & 100.0 \\
\hline
\end{tabular}

near the actual stent. For some stent sizes, ALN was $0 \%$, which may be related to the limited spatial resolution along the SI profile, not allowing detection of subtle changes in apparent lumen diameter.

The Acculink ${ }^{\circledR}$ and Zilver ${ }^{\circledR}$ stents are open-cell, nitinolbased stents. Despite the presence of stent-related artifacts, these stents all showed relatively good lumen visibility with both sequence types, correlating with low ALN $(<25 \%)$ and relatively high RIS $(>75 \%)$ values which were comparable for both CE-MRA and 3D-TOF (Figs. 2 and 3). These results indicate that $3 \mathrm{D}-\mathrm{TOF}$ may be a feasible alternative to CE-MRA in imaging these stents, and in vivo studies of the 3D-TOF sequence may further elucidate its clinical usefulness in follow-up imaging in patients with these stents. In addition, 3D-TOF may be particularly useful in situations where use of gadolinium contrast agents may be harmful or impossible, e.g., in patients with renal insufficiency to avoid the development of complications such as nephrogenic systemic fibrosis. Another open-cell, nitinolbased stent in this study, the Precise ${ }^{\circledR}$, showed considerable loss of in-stent signal (RIS $<45 \%$ with both sequences) and moderate lumen narrowing (ALN <30\%). Although these values are worse than for the Zilver ${ }^{\circledR}$ and Acculink ${ }^{\circledR}$ stents, the results were comparable for both $3 \mathrm{D}-\mathrm{TOF}$ and CE-
Fig. 3 Artificial lumen narrowing. Bars represent ALN values for CE-MRA (dark gray bars) and 3D-TOF (light gray bars) for each stent in the study. Note: ALN was 0 for the Acc8, Acc6, and Pre8 stents

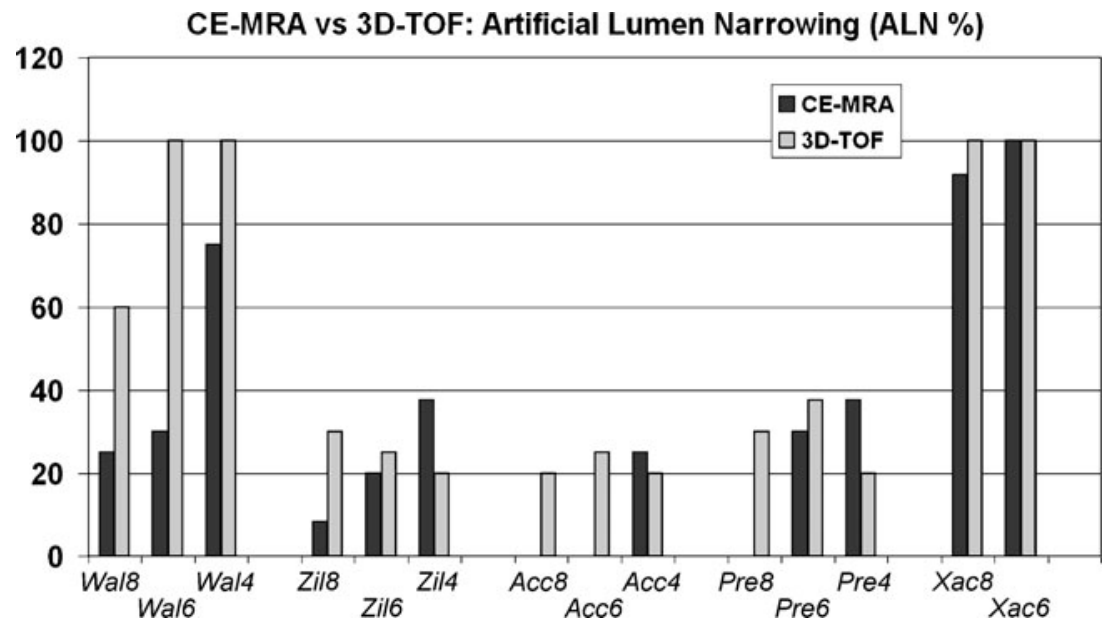


MRA, and image quality was acceptable. Thus, we deem it reasonable to include the Precise ${ }^{\circledR}$ stent in future in vivo evaluations using 3D-TOF or CE-MRA.

The Wallstent ${ }^{\circledR}$ is cobalt alloy based and has a closed cell design. Interestingly, while the larger diameters of this stent offered acceptable image quality with CE-MRA, the results of the 4-mm Wallstent ${ }^{\circledR}$ and the 3D-TOF images of all sizes were less favorable. It remains debatable whether this is related to the stent's geometry (closed cell design with relatively small cell size) or its cobalt alloy material. While previous investigations have shown ALN values as high as $100 \%$ for the Wallstent ${ }^{\circledR}$ [4], in this study, it appears that the Wallstent ${ }^{\circledR}$ may be suitable for imaging with CE-MRA. The reason for this discrepancy may be attributed to a number of factors, including the differences in used "background tissue" (ultrasound gel vs. normal saline), the presence of intravascular flow, or the use of a carotid surface coil. In conclusion, while the previously seen difficulties in imaging the Wallstent ${ }^{\circledR}[4,5]$ were also encountered in this study, it is possible that the use of surface coils in an in vivo setting may lead to clinically useful results with this stent, at least for the detection of significant restenosis $(>70 \%)$.

The other closed cell stent in this study, the Xact ${ }^{\circledR}$, showed an almost complete in-stent signal loss (corresponding to ALN values of up to $100 \%$ ). This confirms the problematic MR characteristics of this stent seen in a previous study with CE-MRA [4]. Although the Xact ${ }^{\circledR}$ stent is made of nitinol, a material commonly associated with favorable MR imaging characteristics $[4,15,16]$, the lumen of the $\mathrm{Xact}^{\circledR}$ was not readily visible in this study. This supports the hypothesis that stent geometry and strut architecture may indeed be at least equally important as stent material in terms of the resulting image quality. However, the influence of the complex stent geometry on stent-related artifacts is difficult to predict at best. Thus, development of stent-specific guidelines for CAS follow-up imaging may be a beneficial approach to overcome this problem. For the development of standard imaging guidelines, different MRA sequence types need to be compared to other available imaging modalities, such as CTA and DSA. For follow-up imaging, MRA has the advantage of included parenchymal stroke imaging. Both CTA and MRA offer information on the bilateral carotid bifurcations, whereas DSA is typically performed on the affected side only. Disadvantages of MRA include relatively high cost as well as limited availability especially of highfield scanners and surface coils.

In vivo studies should be performed to overcome the limitations of the present study: Use of silicone tubes produces artifacts limiting lumen visibility. The straight vessel geometry used probably results in flow patterns differing from in vivo conditions, and artifact patterns may change when imaging flexed or partially rotated stents in tortuous vessels. Stent orientation relative to $B_{0}$ may change under in vivo conditions and was not assessed in this study (all stents were oriented in parallel to $B_{0}$ ). Furthermore, processes such as intimal stent overgrowth may significantly alter stent appearance and must therefore be taken into account [2]. Finally, the objective approach to image analysis used in this study is helpful to accurately compare different modalities or sequence types but is of limited importance in clinical practice. Future studies should also include evaluation of stenosed vessel segments, which may prove especially challenging for the 3D-TOF technique due to stenosis-related turbulence and the possibility of overestimation of stenosis with 3D-TOF.

\section{Conclusion}

Our results show that $3 \mathrm{D}-\mathrm{TOF}$ is a viable alternative to CEMRA for depicting the in-stent lumen in certain carotid artery stents. While both sequence types were limited by stentrelated artifacts, 3D-TOF is non-invasive and offers good image quality with the use of specialized carotid surface coils. 3D-TOF may be particularly useful in situations where use of contrast media may be harmful, such as concomitant renal or thyroid disease. For the Zilver ${ }^{\circledR}$, Acculink ${ }^{\circledR}$, and Precise ${ }^{\circledR}$ stents, 3D-TOF provided relative in-stent signal intensities comparable to CE-MRA. For these stents, further evaluation of 3D-TOF in an in vivo setting appears to be particularly promising. For the Wallstent ${ }^{\circledR}$, both sequence types showed significant stent-related artifacts. For stent diameters $\geq 6 \mathrm{~mm}$, CE-MRA offered acceptable RIS and ALN values with this stent and should be investigated in vivo. The Xact ${ }^{\circledR}$ stent gave strong artifacts with both sequence types and seems not well suited for MR imaging. Thus, stent-specific protocols for follow-up imaging after CAS may be a reasonable option. Specifically, in vivo examinations should carefully investigate the reliability and diagnostic quality of 3D-TOF as compared to CE-MRA and other modalities in light of the results presented here.

Conflict of interest statement We declare that we have no conflict of interest.

Open Access This article is distributed under the terms of the Creative Commons Attribution Noncommercial License which permits any noncommercial use, distribution, and reproduction in any medium, provided the original author(s) and source are credited.

\section{References}

1. Blum MB, Schmook M, Schernthaner R, Edelhauser G, Puchner S, Lammer J, Funovics MA (2007) Quantification and detectability of in-stent stenosis with CT angiography and MR angiography in arterial stents in vitro. AJR Am J Roentgenol 189:1238-1242 
2. Borisch I, Hamer OW, Zorger N, Feuerbach S, Link J (2005) In vivo evaluation of the carotid Wallstent on three-dimensional contrast material-enhanced MR angiography: influence of artifacts on the visibility of stent lumina. J Vasc Interv Radiol 16:669-677

3. Hagspiel KD, Leung DA, Nandalur KR, Angle JF, Dulai HS, Spinosa DJ, Matsumoto AH, Christopher JM, Ahmed H, Berr SS (2005) Contrast-enhanced MR angiography at $1.5 \mathrm{~T}$ after implantation of platinum stents: in vitro and in vivo comparison with conventional stent designs. AJR Am J Roentgenol 184:288-294

4. Hahnel S, Nguyen-Trong TH, Rohde S, Hartmann M, Braun C, Sartor K, Heiland S (2006) 3.0 Tesla contrast-enhanced MR angiography of carotid artery stents: in vitro measurements as compared with 1.5 Tesla. J Neuroradiol 33:75-80

5. Hamer OW, Borisch I, Paetzel C, Nitz WR, Seitz J, Feuerbach S, Zorger N (2006) In vitro evaluation of stent patency and in-stent stenoses in 10 metallic stents using MR angiography. Br J Radiol 79:636-643

6. Klemm T, Duda S, Machann J, Seekamp-Rahn K, Schnieder L, Claussen CD, Schick F (2000) MR imaging in the presence of vascular stents: a systematic assessment of artifacts for various stent orientations, sequence types, and field strengths. J Magn Reson Imaging 12:606-615

7. Maintz D, Kugel H, Schellhammer F, Landwehr P (2001) In vitro evaluation of intravascular stent artifacts in three-dimensional MR angiography. Invest Radiol 36:218-224

8. Straube T, Wolf S, Flesser A, Deli M, Alfke K, Nabavi A, Jansen O (2005) MRI of carotid stents: influence of stent properties and sequence parameters on visualization of the carotid artery lumen. Rofo 177:375-380
9. Wall A, Kugel H, Bachman R, Matuszewski L, Kramer S, Heindel W, Maintz D (2005) $3.0 \mathrm{~T}$ vs. $1.5 \mathrm{~T}$ MR angiography: in vitro comparison of intravascular stent artifacts. J Magn Reson Imaging 22:772-779

10. Agarwal R, Brunelli SM, Williams K, Mitchell MD, Feldman HI, Umscheid CA (2009) Gadolinium-based contrast agents and nephrogenic systemic fibrosis: a systematic review and metaanalysis. Nephrol Dial Transplant 24:856-863

11. Perazella MA (2008) Gadolinium-contrast toxicity in patients with kidney disease: nephrotoxicity and nephrogenic systemic fibrosis. Curr Drug Saf 3:67-75

12. Taylor RA, Qureshi AI (2007) 10 most commonly asked questions about carotid artery stent placement. Neurologist 13:318-322

13. Schoning M, Hartig B (1996) Age dependence of total cerebral blood flow volume from childhood to adulthood. J Cereb Blood Flow Metab 16:827-833

14. Wang Y, Truong TN, Yen C, Bilecen D, Watts R, Trost DW, Prince MR (2003) Quantitative evaluation of susceptibility and shielding effects of nitinol, platinum, cobalt-alloy, and stainless steel stents. Magn Reson Med 49:972-976

15. Lettau M, Sauer A, Heiland S, Rohde S, Reinhardt J, Bendszus M, Hahnel S. (2009) In vitro comparison of different carotid artery stents: a pixel-by-pixel analysis using CT angiography and contrastenhanced MR angiography at 1.5 and $3 \mathrm{~T}$. Neuroradiology (in press)

16. Lettau M, Sauer A, Heiland S, Rohde S, Bendszus M, Hahnel S (2009) Carotid artery stents: in vitro comparison of different stent designs and sizes using CT angiography and contrast-enhanced MR angiography at $1.5 \mathrm{~T}$ and $3 \mathrm{~T}$. AJNR Am J Neuroradiol 30:1993-1997 